\title{
Bioconversion of agro-industrial by-products in rhamnolipids toward applications in enhanced oil recovery and bioremediation
}

\author{
Eduardo J. Gudiña ${ }^{a, *}$, Ana I. Rodrigues ${ }^{a}$, Eliana Alves ${ }^{b}$, M. Rosário Domingues ${ }^{\text {b }}$, José A. Teixeira ${ }^{a}$, \\ Lígia R. Rodrigues ${ }^{\mathrm{a}}$ \\ ${ }^{a}$ CEB - Centre of Biological Engineering, University of Minho, 4710-057 Braga, Portugal \\ ${ }^{\mathrm{b}}$ Mass Spectrometry Centre, QOPNA, Department of Chemistry, University of Aveiro, 3810-193 Aveiro, Portugal
}

\section{H I G H L I G H T S}

- Two agro-industrial by-products were evaluated for biosurfactant production.

- Pseudomonas aeruginosa \#112 produced $3.2 \mathrm{~g}$ biosurfactant/l in the optimized medium.

- The biosurfactant was characterized as a mixture of eight rhamnolipid congeners.

- It exhibited a better performance in oil recovery than two chemical surfactants.

\section{A R T I C L E I N F O}

Article history:

Received 23 September 2014

Received in revised form 12 November 2014

Accepted 15 November 2014

Available online 20 November 2014

\section{Keywords:}

Rhamnolipid

Pseudomonas aeruginosa

Molasses

Corn steep liquor

Bioremediation

\begin{abstract}
A B S T R A C T
In this work, biosurfactant production by a Pseudomonas aeruginosa strain was optimized using low-cost substrates. The highest biosurfactant production $(3.2 \mathrm{~g} / \mathrm{l})$ was obtained using a culture medium containing corn steep liquor $(10 \%(\mathrm{v} / \mathrm{v}))$ and molasses $(10 \%(\mathrm{w} / \mathrm{v}))$. The biosurfactant reduced the surface tension of water up to $30 \mathrm{mN} / \mathrm{m}$, and exhibited a high emulsifying activity ( $E_{24}=60 \%$ ), with a critical micelle concentration as low as $50 \mathrm{mg} / \mathrm{l}$. The biosurfactant produced in this alternative medium was characterized as a mixture of eight different rhamnolipid congeners, being the most abundant the mono-rhamnolipid Rha- $\mathrm{C}_{10}-\mathrm{C}_{10}$. However, using LB medium, nine different rhamnolipid congeners were identified, being the most abundant the di-rhamnolipid Rha-Rha- $C_{10}-C_{10}$. The rhamnolipid mixture produced in the alternative medium exhibited a better performance in removing oil from contaminated sand when compared with two chemical surfactants, suggesting its potential use as an alternative to traditional chemical surfactants in enhanced oil recovery or bioremediation.
\end{abstract}

(c) 2014 Elsevier Ltd. All rights reserved.

\section{Introduction}

Surfactants are an important class of chemical compounds widely used in many different fields. They are amphiphilic molecules that contain distinct hydrophilic and hydrophobic groups, which, by accumulating at the interfaces, reduce the surface or interfacial tension between two phases with different polarities. Most conventional surfactants available are derived from petrochemical sources meaning that they are expensive and hazardous to the environment due to their recalcitrant nature (Vaz et al., 2012).

\footnotetext{
* Corresponding author at: CEB - Centre of Biological Engineering, University of Minho, Campus de Gualtar, 4710-057 Braga, Portugal. Tel.: +351 253 601968; fax: +351253604429.

E-mail address: egudina@deb.uminho.pt (E.J. Gudiña).
}

In the recent years, an increase in environmental awareness has led to much more interest in the use of renewable-based, biodegradable and more environmentally friendly surfactants. The market for these "green" alternatives to synthetic surfactants was 344 kilo tons in 2013, and it is expected to reach 462 kilo tons and 2308 million USD by 2020 (Grand View Research, 2014). Among them, biosurfactants, surface active molecules produced by diverse groups of microorganisms (bacteria, yeasts and filamentous fungi), have attracted considerable attention as alternatives to their synthetic counterparts, owing to their unique properties, that include low toxicity, high biodegradability, high selectivity, low critical micelle concentrations $(\mathrm{cmc})$ and effectiveness at extreme temperatures, pHs and salinities. Due to these properties, biosurfactants comprise a variety of potential applications in agriculture, bioremediation, petrochemical, pharmaceutical, cosmetics, detergent or food industries (Abdel-Mawgoud et al., 2010; Henkel et al., 2012). 
Rhamnolipids (glycolipid biosurfactants) are among the most effective biosurfactants due to their low minimum surface tension values $(28-30 \mathrm{mN} / \mathrm{m})$, high emulsifying activity (emulsifying indexes up to 60-70\%) and low cmcs (10-200 mg/l). Rhamnolipids are usually synthesized by the Gram-negative bacterium Pseudomonas aeruginosa and related species as complex mixtures, and more than 60 different congeners have been identified to date (Abbasi et al., 2012; Abdel-Mawgoud et al., 2010; Benincasa et al., 2004; Bharali and Konwar, 2011). Their hydrophilic moiety consists of one (mono-rhamnolipids) or two (di-rhamnolipids) rhamnose molecules, while the hydrophobic part is represented by two (or more rarely one) $\beta$-hydroxy fatty acids, saturated or unsaturated, of different chain lengths $\left(\mathrm{C}_{8}-\mathrm{C}_{24}\right)$. Their physicochemical properties (e.g., solubility, surface activity) and applications depend on the type and proportions of congeners present in the mixture, which vary according to the bacterial strain, the composition of the culture medium and the cultivation conditions (Abdel-Mawgoud et al., 2010; Raza et al., 2009; Zhang et al., 2014).

Nowadays a limited number of biosurfactants are produced at an industrial scale, mainly due to their high production costs, resulting from the relatively low productivities and the high prices of the culture media used. In order to increase their competitiveness in the market in relation to the chemical surfactants, considerable efforts have been made on the development of economical and sustainable production processes (Benincasa and Accorsini, 2008; Henkel et al., 2012; Sarachat et al., 2010). It is generally accepted that the culture media can account for up to $50 \%$ of the total production cost of biosurfactants (Henkel et al., 2012). Therefore, the use of low-cost renewable feed-stocks and agro-industrial by-products and wastes as culture media can contribute to make their production economically viable and, at the same time, alleviate many industrial waste management problems.

Molasses is a low-cost by-product generated during the crystallization of sugar from liquid extracts of sugarcane or sugar beet. It contains a high concentration of carbohydrates (usually about $50 \%$ ), as well as other valuable compounds such as vitamins. Corn steep liquor (CSL) is a liquid by-product generated by the corn wet milling industry. It is rich in vitamins, minerals, amino acids and proteins, being an important source of nitrogen for many biotechnological processes (Henkel et al., 2012; Maddipati et al., 2011). The high nutritional content of both substrates, together with their availability and low price, make them useful products to be used as culture medium or nutrient supplements for microorganisms in diverse industrial fermentation processes.

The aim of this work was to optimize the biosurfactant production by a $P$. aeruginosa strain isolated from a crude oil sample using low-cost substrates. The biosurfactant mixture produced was characterized, and its applicability in oil recovery was evaluated and compared with two commonly used chemical surfactants.

\section{Methods}

\subsection{Strain and culture conditions}

The biosurfactant-producing strain P. aeruginosa \#112 was isolated from a crude oil sample obtained from a Brazilian oil field, and identified according to its 16S rRNA sequence. The 16S rRNA gene was amplified by PCR using primers $341 \mathrm{~F}$ and $907 \mathrm{R}$. The resulting sequence was compared with those deposited in the GenBank database of the National Center for Biotechnology Information (NCBI) (http://www.ncbi.nlm.nih.gov) using the nucleotidenucleotide blast (BLASTn) network service, to determine its phylogenetic affiliation, and deposited in the GenBank database under the accession number KM244766. The strain was maintained in Luria-Bertani (LB) medium, supplemented with $20 \%(\mathrm{v} / \mathrm{v})$ of glycerol, at $-80^{\circ} \mathrm{C}$. The composition of LB medium was $(\mathrm{g} / \mathrm{l}): \mathrm{NaCl}$ 10.0; tryptone 10.0; yeast extract 5.0; $\mathrm{pH}$ 7.0. Whenever required, frozen stocks were streaked on LB agar plates and incubated at $37^{\circ} \mathrm{C}$ for $16-18 \mathrm{~h}$. The agar plates were stored at $4{ }^{\circ} \mathrm{C}$ no longer than 3 weeks.

\subsection{Chemical surfactants}

Two chemical surfactants, Enordet and Petrostep (kindly provided by SNF FLOERGER (France)), commonly used in chemical enhanced oil recovery, were used to compare its performance with the biosurfactant herein produced by $P$. aeruginosa \#112.

\subsection{Biosurfactant production}

Biosurfactant production by $P$. aeruginosa \#112 was first evaluated in LB medium. $500 \mathrm{ml}$ flasks containing $200 \mathrm{ml}$ of LB medium were inoculated with $2 \mathrm{ml}$ of a pre-culture; pre-cultures were prepared by inoculating $10 \mathrm{ml}$ of LB medium with a single colony taken from an agar plate, and subsequently they were incubated overnight at $37^{\circ} \mathrm{C}$ and $120 \mathrm{rpm}$. The production cultures were also incubated at $37^{\circ} \mathrm{C}$, but at different agitation rates $(120,180$ and $200 \mathrm{rpm})$. Samples were taken along the fermentation to determine biosurfactant production. The samples were centrifuged $\left(10,000 \times \mathrm{g}, 20 \mathrm{~min}, 20^{\circ} \mathrm{C}\right)$ and the cell-free supernatants were used to measure the surface tension (ST) and to determine the emulsifying activity $\left(E_{24}\right)$, as described below. Whenever required, the culture broth supernatants were diluted 10 or 100 times with demineralized water, and the surface tension $\left(\mathrm{ST}^{-1}, \mathrm{ST}^{-2}\right)$ and emulsifying activities $\left(E_{24}^{-1}, E_{24}^{-2}\right)$ were measured.

\subsection{Use of alternative substrates for the biosurfactant production}

Sugarcane molasses (kindly provided by RAR: Refinarias de Açúcar Reunidas, S.A. (Portugal)) and CSL (kindly provided by COPAM: Companhia Portuguesa de Amidos, S.A. (Portugal)) were evaluated as alternative substrates for biosurfactant production by $P$. aeruginosa \#112. Molasses contained $490 \mathrm{~g} / \mathrm{l}$ of carbohydrates and $0.6 \mathrm{~g} / \mathrm{l}$ of protein, whereas CSL contained $75 \mathrm{~g} / \mathrm{l}$ of carbohydrates and $5 \mathrm{~g} / \mathrm{l}$ of protein. Total carbohydrates and protein concentrations were determined using the phenol-sulfuric and Lowry methods, respectively (Dubois et al., 1956; Lowry et al., 1951). Molasses was dissolved in demineralized water $(10 \%(\mathrm{w} / \mathrm{v}))$ and used as culture medium, either alone or supplemented with different nitrogen sources at a concentration of $2 \mathrm{~g} / \mathrm{l}$. The nitrogen sources used were: ammonium nitrate, ammonium sulfate, sodium nitrate, tryptone, urea and yeast extract. CSL was dissolved in demineralized water at different concentrations (5\%,10\% and 15\% (v/v)) and used as culture medium, either alone or supplemented with glucose at a concentration of $10 \mathrm{~g} / \mathrm{l}$. All media were adjusted to $\mathrm{pH}$ 7.0. The assays were performed in $500 \mathrm{ml}$ flasks containing $200 \mathrm{ml}$ of the different media. Each flask was inoculated with $2 \mathrm{ml}$ of a pre-culture grown in LB medium for $24 \mathrm{~h}$. The cultures were incubated at $37^{\circ} \mathrm{C}$ and $180 \mathrm{rpm}$. Samples were taken at different time points during the fermentation to determine the biosurfactant production as described above.

\subsection{Biosurfactant recovery}

The biosurfactant produced by $P$. aeruginosa \#112 was recovered from the cell-free supernatants obtained at the end of the different fermentations by adsorption chromatography using the polystyrene resin Amberlite XAD-2 (Sigma-Aldrich). A $125 \mathrm{ml}$ column was filled with Amberlite XAD-2 and equilibrated with three volumes of $0.1 \mathrm{M}$ potassium phosphate buffer $(\mathrm{pH} 6.1)$ at a constant flow rate of $3 \mathrm{ml} / \mathrm{min}$. The cell-free supernatants were 
adjusted to $\mathrm{pH} 6.1$ and introduced into the column at the same flow rate. Subsequently, the column was washed with three volumes of demineralized water to remove the non-adsorbed compounds. Finally, the biosurfactant adsorbed to the resin was eluted with three volumes of methanol; the methanol was removed using a rotary evaporator at room temperature and the crude biosurfactant was dissolved in a minimal amount of demineralized water and freeze-dried. The products obtained were weighed and stored at $-20^{\circ} \mathrm{C}$ for further studies.

\subsection{Surface-activity determination}

Surface tension measurements of biosurfactant solutions and culture broth supernatants were performed according to the Ring method described elsewhere (Gudiña et al., 2010) using a KRÜSS K6 Tensiometer (KRÜSS GmbH, Germany) equipped with a $1.9 \mathrm{~cm}$ De Noüy platinum ring at room temperature $\left(25^{\circ} \mathrm{C}\right)$. All measurements were performed in triplicate.

\subsection{Emulsifying activity determination}

The emulsifying activity of the cell-free supernatants and biosurfactant solutions was determined using $n$-hexadecane, as described elsewhere (Vaz et al., 2012). All emulsification indexes were determined in triplicate.

\subsection{Critical micelle concentration $(\mathrm{cmc})$}

Critical micelle concentration, the concentration of an amphiphilic compound in solution at which the formation of micelles is initiated, was calculated as described elsewhere (Gudiña et al., 2010), using the freeze-dried biosurfactant produced by P. aeruginosa \#112 dissolved in demineralized water at different concentrations.

\subsection{Biosurfactant chemical characterization}

For chemical composition analysis, the biosurfactant mixtures were extracted using the Folch extraction method commonly used to extract lipids from biomolecules (Folch et al., 1957). Briefly, the freeze-dried biosurfactants were dissolved in demineralized water at a concentration of $10 \mathrm{mg} / \mathrm{ml}$. Afterwards, a chloroform/methanol mixture $(2: 1, v / v)$ was added to the biosurfactant solution, achieving a final chloroform/methanol/water ratio of $8: 4: 3(\mathrm{v} / \mathrm{v} /$ v). After mixing, the mixture was centrifuged $(9000 \times \mathrm{g}, 5 \mathrm{~min})$, the organic layer was collected and the samples were evaporated to dryness by vacuum at room temperature; once dried, the samples were stored at $-20^{\circ} \mathrm{C}$. Prior to analysis, the samples were re-dissolved in $100 \mu \mathrm{l}$ of chloroform.

Lipid extracts were analyzed by electrospray ionization (ESI) mass spectrometry (MS) using a quadrupole time-of-flight ( $Q$ TOF) mass spectrometer (Micromass, Manchester, UK) and a LXQ linear ion trap mass spectrometer (Thermo Scientific, San Jose, CA, USA). ESI-MS and MS/MS conditions in the ESI-Q-TOF mass spectrometer were as follows: electrospray voltage was $3 \mathrm{kV}$ in the positive ion mode with a cone voltage of $30 \mathrm{~V}$. The source temperature was $80^{\circ} \mathrm{C}$ and the desolvation temperature was $150^{\circ} \mathrm{C}$. Collision energy used for the MS/MS spectra varied between 30 and $40 \mathrm{~V}$. Data acquisition and treatment of results were carried out with MassLynx ${ }^{\mathrm{TM}}$ software V4.0 (Waters). ESI-MS and MS/MS conditions obtained on a Finnigan LXQ linear ion trap mass spectrometer were as follows: electrospray voltage $5 \mathrm{kV}$; capillary temperature $275^{\circ} \mathrm{C}$, and the sheath gas (He) flow rate 25 units. The normalized collision energy (CE) varied between 19 and 21 (arbitrary units) for MS/MS. Data acquisition and treatment of results were carried out with the XcaliburW Data System 2.0 (Thermo Scientific, San Jose, CA, USA).

\subsection{Application of (bio)surfactants in the removal of crude oil from sand}

The applicability of the biosurfactant mixture produced by $P$. aeruginosa \#112 and the chemical surfactants Enordet and Petrostep in oil recovery was studied using an artificially contaminated sand containing $10 \%(\mathrm{w} / \mathrm{w})$ of Arabian Light crude oil (kindly provided by GALP, Portugal). The assays were performed in triplicate, at $40^{\circ} \mathrm{C}$, as described by Pereira et al. (2013).

\section{Results and discussion}

\subsection{Biosurfactant production by P. aeruginosa \#112 in LB medium}

Biosurfactant production by $P$. aeruginosa \#112 was studied using LB medium at $37^{\circ} \mathrm{C}$ with different agitation rates: 120,180 and $200 \mathrm{rpm}$. The results obtained at 120 and $180 \mathrm{rpm}$ are shown in Fig. 1.

As it can be seen from the results obtained, the biosurfactant produced by $P$. aeruginosa \#112 reduced the culture medium surface tension to values around $33 \mathrm{mN} / \mathrm{m}$, with emulsifying indexes against $n$-hexadecane as high as $60 \%$. Taking into account that to calculate the emulsifying activity, the same volume of cell-free supernatant and $n$-hexadecane was used, emulsifying indexes
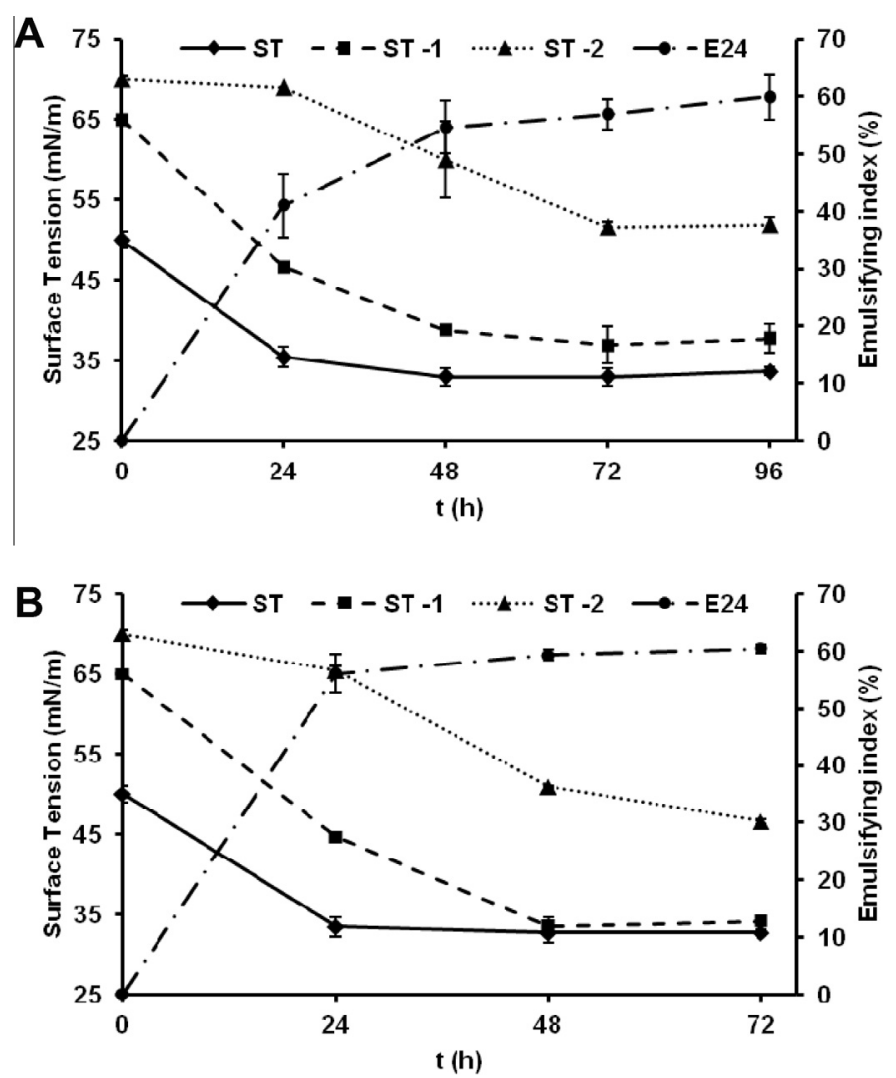

Fig. 1. Surface tension values $(\mathrm{mN} / \mathrm{m})$ and emulsifying indexes (\%) obtained with Pseudomonas aeruginosa \#112 grown in LB medium at $37^{\circ} \mathrm{C}$ and 120 (A) or 180 (B) $\mathrm{rpm}$. ST, $\mathrm{ST}^{-1}$ and $\mathrm{ST}^{-2}$ represent the surface tension values of the cell-free supernatants without dilution, 10 times diluted and 100 times diluted (with demineralized water), respectively. The ST, $\mathrm{ST}^{-1}$ and $\mathrm{ST}^{-2}$ values of $\mathrm{LB}$ medium were $50.0 \pm 0.5,65.0 \pm 0.3$ and $70.0 \pm 0.5 \mathrm{mN} / \mathrm{m}$, respectively. Results represent the average of three independent experiments \pm standard deviation. 
higher than $50 \%$ entail that the oil phase was completely emulsified.

After $24 \mathrm{~h}$ of growth, both at 120 and $180 \mathrm{rpm}$, the surface tension of the cell-free supernatants remained nearly constant until the end of the fermentation. This means that the amount of biosurfactant present in the medium was equal or higher than the $\mathrm{cmc}$. In those conditions it was necessary to dilute the culture broth supernatants to study the evolution of biosurfactant production. As it can be seen from Fig. 1, the $\mathrm{ST}^{-1}$ and $\mathrm{ST}^{-2}$ values continued decreasing after $24 \mathrm{~h}$, meaning that biosurfactant production continued until the end of the fermentation.

According to the $\mathrm{ST}^{-1}$ and $\mathrm{ST}^{-2}$ values, the incubation at $180 \mathrm{rpm}$ offered better results when compared with $120 \mathrm{rpm}$ (evolution of biosurfactant production was faster and a higher decrease in surface tension was achieved). Experiments performed at $200 \mathrm{rpm}$ resulted in similar surface tension values when compared with those obtained at $180 \mathrm{rpm}$ (data not shown). At $120 \mathrm{rpm}$, the amount of biosurfactant recovered at the end of the fermentation was $1067 \pm 64 \mathrm{mg} / \mathrm{l}$, whereas at $180 \mathrm{rpm}$ it was $1472 \pm 83 \mathrm{mg} / \mathrm{l}$, which is in agreement with the surface tension values obtained in both tested conditions. The $\mathrm{cmc}$ of this biosurfactant was found to be $90 \mathrm{mg} / \mathrm{l}$, with a minimum surface tension value of $29.8 \pm 0.1 \mathrm{mN} / \mathrm{m}$.

The results obtained are in agreement with the general notion on this point in the literature. It has been reported that rhamnolipid production by $P$. aeruginosa is inefficient under oxygen-limiting conditions, and as the agitation rates increase (up to 150$250 \mathrm{rpm}$ ), rhamnolipid production also increases due to a higher dissolved oxygen concentration in the medium (Abbasi et al., 2012; Abdel-Mawgoud et al., 2009; Aparna et al., 2012; Bharali and Konwar, 2011; Cha et al., 2008; Haba et al., 2003; Lotfabad et al., 2009, 2010; Pacheco et al., 2012; Wei et al., 2005).

\subsection{Use of alternative substrates for biosurfactant production}

In order to reduce the production costs, molasses $(10 \%(\mathrm{w} / \mathrm{v}))$ and molasses supplemented with different nitrogen sources (at a concentration of $2 \mathrm{~g} / \mathrm{l}$ ) were used as culture medium. As it can be seen from the results gathered in Table 1, in all cases the surface tension values $(29.2-30.8 \mathrm{mN} / \mathrm{m})$ were generally lower than those obtained with LB medium. Differences between the different media can be observed comparing the $\mathrm{ST}^{-1}$ and $\mathrm{ST}^{-2}$ values, as well as the emulsifying activities. The less favorable result was obtained using molasses without any additional nitrogen source, as can be confirmed by the low amount of biosurfactant produced $(499 \pm 82 \mathrm{mg} / \mathrm{l})$ and the lack of emulsifying activity. Using ammonium nitrate, ammonium sulfate, sodium nitrate or urea as nitrogen sources, similar results were obtained regarding the surface tension values. The highest emulsifying activity $(58.0 \pm 2.3 \%)$ was achieved using urea as the nitrogen source, whereas the lowest emulsifying indexes were obtained with tryptone and yeast extract. Among all media evaluated, the amount of biosurfactant produced (580-888 $\mathrm{mg} / \mathrm{l}$ ) was lower than that obtained with LB medium at the same conditions.

In other set of experiments, CSL at different concentrations (5\%, $10 \%$ and $15 \%(\mathrm{v} / \mathrm{v}))$ was used as culture medium, either alone or supplemented with glucose $(10 \mathrm{~g} / \mathrm{l})$ or molasses $(10 \%(\mathrm{w} / \mathrm{v}))$. The cultures were performed at the same conditions previously described $\left(37^{\circ} \mathrm{C}\right.$ and $180 \mathrm{rpm}$ ). The best results regarding biosurfactant production were obtained using a medium containing CSL $(10 \%(\mathrm{v} / \mathrm{v}))$ and molasses $(10 \%(\mathrm{w} / \mathrm{v}))$. Evolution of surface tension and emulsifying activity with this medium (CSLM) is illustrated in Fig. 2.

Using CSLM, the surface tension values obtained $\left(\mathrm{ST}^{-2}=38.7 \pm\right.$ $2.3 \mathrm{mN} / \mathrm{m}$ ) were lower when compared with the ones obtained with the previous media, thus suggesting a higher biosurfactant production. Also the emulsifying activity was higher; similar emulsifying indexes were obtained with the culture broth supernatants without dilution and 10 times diluted (about 60\%), and even the 100 times diluted supernatants exhibited an emulsifying activity close to $30 \%$. Emulsifying indexes between $50 \%$ and $75 \%$ have been reported for different rhamnolipid mixtures by other authors (Abbasi et al., 2012; Abdel-Mawgoud et al., 2009; Aparna et al., 2012; Benincasa and Accorsini, 2008; Bharali and Konwar, 2011; George and Jayachandran, 2009; Lotfabad et al., 2009; Wei et al., 2005). The ability to form and stabilize emulsions is one of the most important features to be considered for the practical application of biosurfactants, mainly for environmental applications such as bioremediation and enhanced oil recovery (Vaz et al., 2012).

The amount of biosurfactant recovered at the end of the fermentation $(144 \mathrm{~h}$ ) was $3194 \pm 245 \mathrm{mg} / \mathrm{l}$, i.e. about two times the amount of biosurfactant produced in LB medium. Furthermore, the $\mathrm{cmc}$ of this biosurfactant was $50 \mathrm{mg} / \mathrm{l}$, meaning that it is more efficient when compared with the one produced in LB medium. Patel and Desai (1997) reported the biosurfactant production by a $P$. aeruginosa strain using a medium containing molasses $(7 \%$ $(\mathrm{v} / \mathrm{v}))$ and $\operatorname{CSL}(0.5 \%(\mathrm{v} / \mathrm{v}))$, although in that case, the amount of biosurfactant produced $(240 \mathrm{mg} / \mathrm{l})$ was considerably lower.

The promising future of biosurfactants appears to depend on the use of abundant low-cost raw materials, and the optimization of the culture conditions to achieve high production yields. Different substrates have been used to produce rhamnolipids, including orange peel (George and Jayachandran, 2009), olive oil (Wei et al., 2005), coconut oil (George and Jayachandran, 2013), wastes from the sunflower-oil production process (Benincasa and Accorsini, 2008; Benincasa et al., 2004), waste frying oil (Haba et al., 2003), soybean oil (Abbasi et al., 2012; Cha et al., 2008; Lotfabad et al., 2010) or molasses (Aparna et al., 2012), with productivities between 3.5 and $12 \mathrm{~g}$ of biosurfactant per liter. However, it has to be taken into consideration that in some of these works, the biosurfactant concentration was calculated indirectly as rhamnose concentration (Benincasa and Accorsini, 2008; Benincasa et al., 2004; George and Jayachandran, 2009, 2013; Haba et al., 2003). Perfumo et al. (2013) reported that the orcinol assay, a method

Table 1

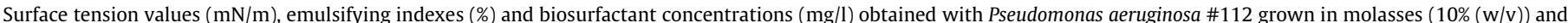

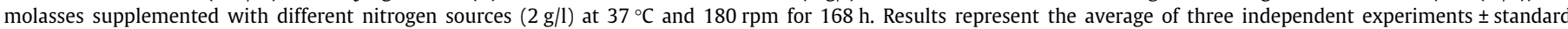

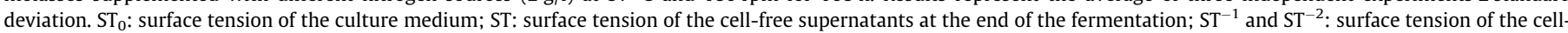
free supernatants at the end of the fermentation diluted 10 and 100 times with demineralized water, respectively; $E_{24}$ : emulsifying index; BS: biosurfactant concentration.

\begin{tabular}{|c|c|c|c|c|c|c|}
\hline & $\mathrm{ST}_{0}(\mathrm{mN} / \mathrm{m})$ & $\mathrm{ST}(\mathrm{mN} / \mathrm{m})$ & $\mathrm{ST}^{-1}(\mathrm{mN} / \mathrm{m})$ & $\mathrm{ST}^{-2}(\mathrm{mN} / \mathrm{m})$ & $E_{24}(\%)$ & $\mathrm{BS}(\mathrm{mg} / \mathrm{l})$ \\
\hline Molasses & $53.4 \pm 0.8$ & $29.9 \pm 0.1$ & $39.4 \pm 0.5$ & $58.0 \pm 4.4$ & $0.0 \pm 0.0$ & $499 \pm 82$ \\
\hline Molasses $+\mathrm{NH}_{4} \mathrm{NO}_{3}$ & $54.4 \pm 0.2$ & $30.5 \pm 0.4$ & $34.9 \pm 1.0$ & $49.8 \pm 3.6$ & $50.0 \pm 2.8$ & $888 \pm 43$ \\
\hline Molasses $+\mathrm{NaNO}_{3}$ & $54.8 \pm 0.4$ & $30.2 \pm 0.1$ & $34.3 \pm 0.9$ & $48.6 \pm 3.5$ & $52.0 \pm 5.7$ & $781 \pm 52$ \\
\hline Molasses $+\left(\mathrm{NH}_{4}\right)_{2} \mathrm{SO}_{4}$ & $55.2 \pm 0.8$ & $30.3 \pm 0.3$ & $34.8 \pm 0.9$ & $49.1 \pm 1.5$ & $53.0 \pm 2.0$ & $888 \pm 24$ \\
\hline Molasses + tryptone & $53.0 \pm 0.5$ & $29.2 \pm 0.3$ & $34.2 \pm 1.1$ & $53.0 \pm 3.8$ & $18.7 \pm 4.6$ & $822 \pm 40$ \\
\hline Molasses + yeast Extract & $54.7 \pm 0.9$ & $29.3 \pm 0.2$ & $36.5 \pm 0.7$ & $55.6 \pm 1.9$ & $37.3 \pm 4.6$ & $742 \pm 51$ \\
\hline Molasses + urea & $54.1 \pm 1.4$ & $30.8 \pm 0.2$ & $36.0 \pm 0.3$ & $50.6 \pm 1.8$ & $58.0 \pm 2.3$ & $580 \pm 37$ \\
\hline
\end{tabular}



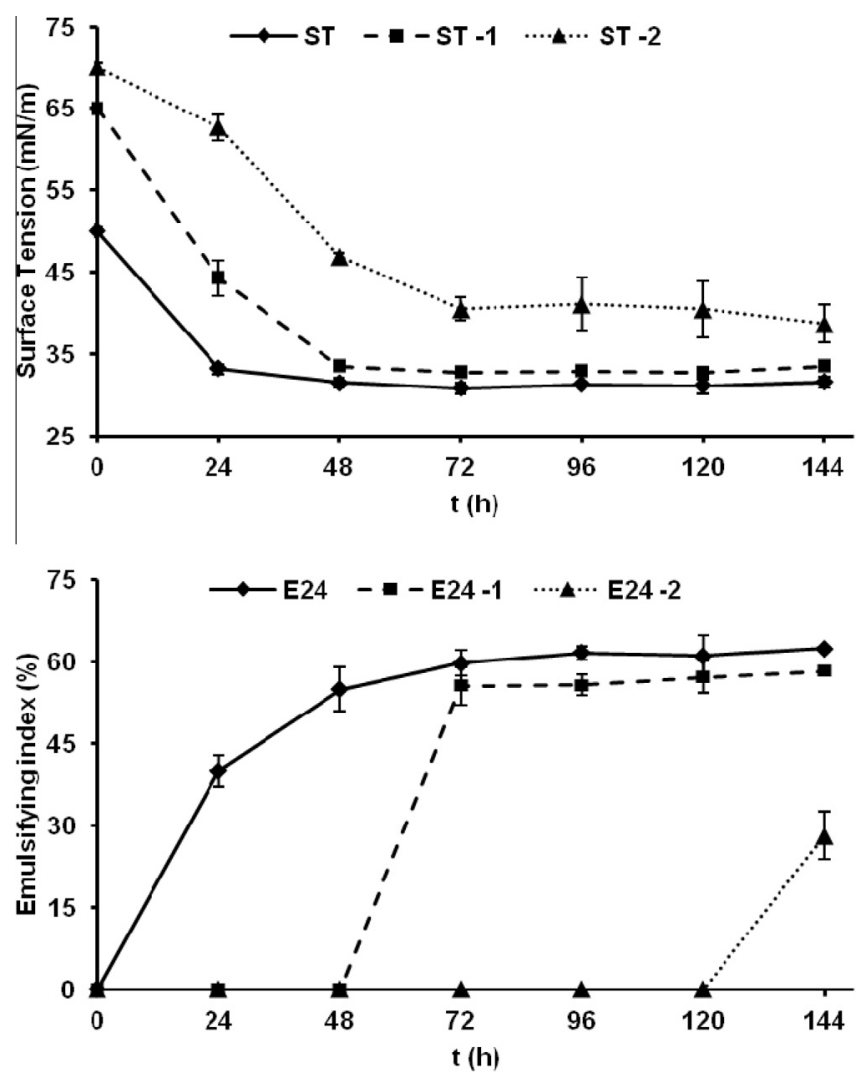

Fig. 2. Surface tension values $(\mathrm{mN} / \mathrm{m})$ and emulsifying indexes (\%) obtained with Pseudomonas aeruginosa \#112 grown in CSLM at $37{ }^{\circ} \mathrm{C}$ and $180 \mathrm{rpm}$ for $144 \mathrm{~h}$. ST/ $E_{24}, \mathrm{ST}^{-1} / E_{24}^{-1}$ and $\mathrm{ST}^{-2} / E_{24}^{-2}$ represent the surface tension values/emulsifying indexes of the cell-free supernatants without dilution, 10 times diluted and 100 times diluted (with demineralized water), respectively. The ST, ST ${ }^{-1}$ and $\mathrm{ST}^{-2}$ values of CSLM medium were $50.0 \pm 0.2,65.0 \pm 0.2$ and $70.0 \pm 0.5 \mathrm{mN} / \mathrm{m}$, respectively. Results represent the average of three independent experiments \pm standard deviation.

commonly used to quantify rhamnolipids, overestimates (between 5 and 9 times) the real rhamnolipid concentration in the samples, probably due to interferences with other compounds. Furthermore, in some of these works, the culture media were supplemented with additional nitrogen sources (e.g. peptone or yeast extract) (Aparna et al., 2012; George and Jayachandran, 2009, 2013), which increases the production costs. Finally, $P$. aeruginosa strains usually only produce considerable amounts of rhamnolipids when hydrocarbons or vegetable oils are used as carbon sources, whereas the use of water soluble substrates results in low yields, which limit their production at large-scale (Abbasi et al., 2012; Benincasa and Accorsini, 2008; Benincasa et al., 2004; Bharali and Konwar, 2011; Cha et al., 2008; George and Jayachandran, 2013; Haba et al., 2003; Wei et al., 2005). In the present work, a high biosurfactant production was achieved using a culture medium containing a combination of two low-cost agro-industrial by-products, without addition of salts, micronutrients or other supplements that would raise the price of the production process (the estimated price of CSLM is $0.024 € / 1$ ), and without the addition of water-immiscible substrates, which makes easier the fermentation and recovery processes.

\subsection{Biosurfactants chemical characterization}

The mass spectrometry analysis of biosurfactant mixtures produced by $P$. aeruginosa \#112 in LB and CSLM medium revealed a very similar profile in their composition. Mixtures of eight and nine rhamnolipid congeners (in CSLM and LB medium, respectively) were identified, and confirmed by the presence of $[\mathrm{M}+\mathrm{Na}]^{+}$ions at $m / z 499.3,523.3,527.3,553.3,555.4,645.3,673.3,699.3$ and 701.4 observed in the electrospray mass spectra (ESI-MS). The structures of the mono- and di-rhamnolipids were confirmed by electrospray tandem mass spectrometry (ESI-MS/MS) as previously reported (Pereira et al., 2012). The different molecular ions detected and the corresponding rhamnolipid congeners are listed in Table 2. The main difference between both media was observed in the relative abundance of the different congeners. In LB medium, the most abundant congener was the di-rhamnolipid Rha-Rha- $\mathrm{C}_{10^{-}}$ $\mathrm{C}_{10}$ (ion at $m / z$ 673.3), whereas in CSLM, the most abundant was the mono-rhamnolipid Rha- $\mathrm{C}_{10}-\mathrm{C}_{10}$ (ion at $\mathrm{m} / z$ 527.3) (Fig. 3).

Rhamnolipids are produced by $P$. aeruginosa strains as mixtures of different congeners, being the most common L-rhamnosyl- $\beta$ hydroxydecanoyl- $\beta$-hydroxydecanoate $\left(\right.$ Rha- $\left.C_{10}-C_{10}\right)$ and Lrhamnosyl-L-rhamnosyl- $\beta$-hydroxydecanoyl- $\beta$-hydroxydecanoate (Rha-Rha- $\mathrm{C}_{10}-\mathrm{C}_{10}$ ). Other congeners frequently found include mono- and di-rhamnolipids with acyl chains containing 8, 10, 12 or 14 carbons, mostly saturated, and, less often, containing one or two double bonds, as well as with only one $\beta$-hydroxy fatty acid (Abbasi et al., 2012; Aparna et al., 2012; Benincasa and Accorsini, 2008; Benincasa et al., 2004; Bharali and Konwar, 2011; George and Jayachandran, 2013; Haba et al., 2003; Lotfabad et al., 2010; Pereira et al., 2012; Zhang et al., 2014). Exceptionally, acyl chains with a higher $\left(\mathrm{C}_{18}, \mathrm{C}_{22}\right.$ and $\left.\mathrm{C}_{24}\right)$ or an odd number of carbons can be found (Hosková et al., 2013; Nie et al., 2010).

The surface-active properties of rhamnolipids are affected by the size of their hydrophilic head (one or two rhamnoses), the length of the alkyl chains and the presence of double bonds. These properties affect the stability of rhamnolipids in the aqueous phase or their capability to solubilize hydrophobic organic compounds (Abbasi et al., 2012; Benincasa et al., 2004; Bharali and Konwar, 2011). Mono-rhamnolipids are less soluble in water and adsorb to surfaces strongly than di-rhamnolipids. More hydrophilic congeners (Rha-Rha- $\mathrm{C}_{10}$, Rha-Rha- $\mathrm{C}_{10}-\mathrm{C}_{10}$ ) exhibit higher $\mathrm{cmcs}$ (200 mg/ 1 ), whereas more hydrophobic ones (Rha- $C_{10}-C_{10}$, or with longer acyl chains) show lower cmcs (between 5 and $40 \mathrm{mg} / \mathrm{l}$ ) and reduce the surface tension more efficiently. The cmcs of rhamnolipids containing unsaturated hydrophobic chains are higher than those of the corresponding saturated forms, because the introduction of unsaturations into the hydrophobic groups affects the packing and organization of biosurfactant molecules at the interface

Table 2

Rhamnolipid congeners identified as $[\mathrm{M}+\mathrm{Na}]^{+}$ions observed in the ESI-MS spectra obtained in the ESI-Q-TOF-mass spectrometer of lipid extracts of biosurfactant mixtures produced by Pseudomonas aeruginosa \#112 in LB and CSLM medium.

\begin{tabular}{llcc}
\hline Rhamnolipid congeners & $m / z[\mathrm{M}+\mathrm{Na}]^{+}$ & LB & CSLM \\
\hline Mono-rhamnolipids & & & \\
Rha- $\left(\mathrm{C}_{10}-\mathrm{C}_{8}\right)^{\mathrm{a}}$ & 499.3 & + & + \\
Rha- $\left(\mathrm{C}_{10: 1}-\mathrm{C}_{10: 1}\right)$ & 523.3 & + & - \\
Rha- $\left(\mathrm{C}_{10}-\mathrm{C}_{10}\right)$ & 527.3 & + & $+^{*}$ \\
Rha- $\left(\mathrm{C}_{10}-\mathrm{C}_{12: 1}\right)^{\mathrm{b}}$ & 553.3 & + & + \\
Rha- $\left(\mathrm{C}_{10}-\mathrm{C}_{12}\right)^{\mathrm{c}}$ & 555.4 & + & + \\
Di-rhamnolipids & & & + \\
Rha-Rha- $\left(\mathrm{C}_{8-} \mathrm{C}_{10}\right)^{\mathrm{d}}$ & 645.3 & + & + \\
Rha-Rha- $\left(\mathrm{C}_{10}-\mathrm{C}_{10}\right)$ & 673.3 & $+^{*}$ & + \\
Rha-Rha- $\left(\mathrm{C}_{10}-\mathrm{C}_{12: 1}\right)^{\mathrm{e}}$ & 699.3 & + & + \\
Rha-Rha- $\left(\mathrm{C}_{10}-\mathrm{C}_{12}\right)^{\mathrm{f}}$ & 701.4 & + & + \\
\hline
\end{tabular}

* Most abundant ion.

a Or Rha- $\left(\mathrm{C}_{8}-\mathrm{C}_{10}\right)$.

b Or Rha- $\left(\mathrm{C}_{12: 1}-\mathrm{C}_{10}\right)$.

c Or Rha- $\left(\mathrm{C}_{12}-\mathrm{C}_{10}\right)$.

d Or Rha-Rha- $\left(\mathrm{C}_{10}-\mathrm{C}_{8}\right)$.

e Or Rha-Rha- $\left(C_{10: 1}-C_{12}\right)$ or Rha-Rha- $\left(C_{12: 1}-C_{10}\right)$ or Rha-Rha- $\left(C_{12}-C_{10: 1}\right)$.

f Or Rha-Rha- $\left(\mathrm{C}_{12}-\mathrm{C}_{10}\right)$. 

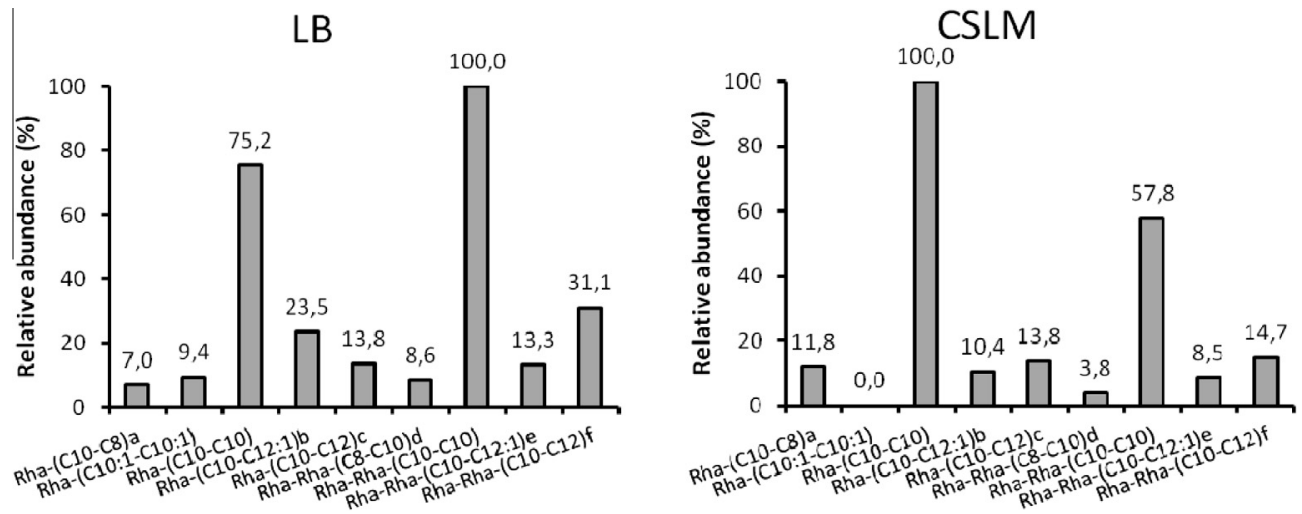

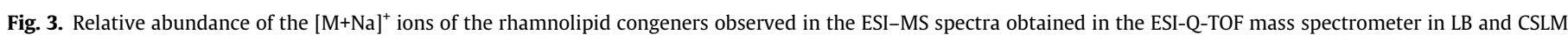
medium.

(Abdel-Mawgoud et al., 2009; Bharali and Konwar, 2011; Haba et al., 2003; Sarachat et al., 2010; Zhang et al., 2014).

The composition and distribution of rhamnolipid congeners vary according to the bacterial strain, the culture conditions and the media composition. Even using the same strain, the culture medium used can influence the composition of the rhamnolipid mixture (Benincasa and Accorsini, 2008; Raza et al., 2009). In the case of $P$. aeruginosa \#112, using LB or CSLM medium, almost the same rhamnolipid congeners were produced. However, using LB medium, the most abundant congener was the di-rhamnolipid Rha-Rha- $\mathrm{C}_{10}-\mathrm{C}_{10}$, whereas using CSLM, the most abundant was the mono-rhamnolipid Rha- $\mathrm{C}_{10}-\mathrm{C}_{10}$, which explains the differences observed in the cmcs of both biosurfactant mixtures.

\subsection{Application of (bio)surfactants in the removal of crude oil from sand}

The applicability of the rhamnolipid mixture produced by $P$. aeruginosa \#112 in CSLM medium and the chemical surfactants Enordet and Petrostep in oil recovery was evaluated. Flasks containing crude oil-contaminated sand were incubated with biosurfactant and chemical surfactants solutions at different concentrations. The percentages of oil recovered at $40{ }^{\circ} \mathrm{C}$ are presented in Table 3.

As it can be seen from the results obtained, the biosurfactant mixture and the chemical surfactant Enordet exhibited similar performances in oil recovery. However, the biosurfactant proved to be more efficient than the chemical surfactant Petrostep. The recovery obtained with the rhamnolipid mixture at a concentration of $1 \mathrm{mg} /$ $\mathrm{ml}(22.1 \pm 2.5 \%)$ was similar to the obtained by Pereira and co-workers (2013) using the lipopeptide biosurfactants produced

\section{Table 3}

Percentages of oil recovered using the biosurfactant mixture produced by Pseudomonas aeruginosa \#112 in CSLM and the chemical surfactants Enordet and Petrostep at different concentrations. Results represent the average of three independent experiments \pm standard deviation.

\begin{tabular}{lll}
\hline (Bio)surfactant & Concentration $(\mathrm{mg} / \mathrm{ml})$ & Oil removed $(\%)$ \\
\hline P. aeruginosa \#112 & 5.0 & $55.0 \pm 3.4$ \\
& 2.5 & $43.7 \pm 0.8$ \\
Enordet & 1.0 & $22.1 \pm 2.5$ \\
& 5.0 & $54.4 \pm 5.9$ \\
& 2.5 & $49.1 \pm 1.7$ \\
Petrostep & 1.0 & $23.9 \pm 1.7$ \\
& 5.0 & $30.5 \pm 2.5$ \\
& 2.5 & $25.7 \pm 4.2$ \\
Control & 1.0 & $15.6 \pm 1.7$ \\
\hline
\end{tabular}

by three Bacillus subtilis strains at the same concentration (18.8$22.1 \%)$.

The same assays were also performed using the cell-free supernatant obtained after growing $P$. aeruginosa \#112 in CSLM medium for $144 \mathrm{~h}$. The percentage of oil recovered in this case $(64.2 \pm 3.5 \%)$ was higher than the obtained with the highest biosurfactant concentration tested $(5 \mathrm{mg} / \mathrm{ml})$. Since the biosurfactant concentration in the cell-free supernatant was about $3.2 \mathrm{mg} / \mathrm{ml}$, the higher oil recovery observed can be due to the effect of other metabolites present in the supernatants. The possibility of using the cell-free supernatants without further processing steps can contribute to increase the use of biosurfactants in applications such as enhanced oil recovery or bioremediation, as their purification constitutes a relevant portion of the overall production costs.

\section{Conclusions}

In this work, a low-cost culture medium was developed using two agro-industrial by-products (corn steep liquor and molasses) for biosurfactant production by a $P$. aeruginosa strain. The amount of biosurfactant produced with this alternative medium was two times the amount of biosurfactant produced in LB medium. This biosurfactant was characterized as a mixture of eight different rhamnolipid congeners, and exhibited a better performance in oil recovery assays when compared with the chemical surfactants Enordet and Petrostep, making it a promising candidate for application in the oil industry or bioremediation processes.

\section{Acknowledgements}

The authors acknowledge the financial support from the Strategic Project PEst-OE/EQB/LA0023/2013, NORTE-07-0124FEDER-000028, NORTE-07-0124-FEDER-000027, QOPNA research unit (project PEst-C/QUI/UI0062/2013; FCOMP-01-0124-FEDER037296) and the Portuguese National Mass Spectrometry Network, RNEM (REDE/1504/REM/2005) funded by Fundação para a Ciência e a Tecnologia (FCT, Portugal), and from AdI (Agência de Inovação, S.A.), through the project BIOCLEAN - "Development of biosurfactant-based products for surfaces cleaning and disinfection in the food industry" (QREN Ref. 30215). Eliana Alves is also grateful to FCT for her post-doctoral grant (BPD/UI51/5441/2014) supported by the project REDE/1504/REM/2005.

\section{Appendix A. Supplementary data}

Supplementary data associated with this article can be found, in the online version, at http://dx.doi.org/10.1016/j.biortech.2014.11. 069. 


\section{References}

Abbasi, H., Hamedi, M.M., Lotfabad, T.B., Zahiri, H.S., Sharafi, H., Masoomi, F., Moosavi-Movahedi, A.A., Ortiz, A., Amanlou, M., Noghabi, K.A., 2012. Biosurfactant-producing bacterium, Pseudomonas aeruginosa MA01 isolated from spoiled apples: physicochemical and structural characteristics of isolated biosurfactant. J. Biosci. Bioeng. 113, 211-219.

Abdel-Mawgoud, A.M., Aboulwafa, M.M., Hassouna, N.A.H., 2009. Characterization of rhamnolipid produced by Pseudomonas aeruginosa isolate Bs20. Appl. Biochem. Biotechnol. 157, 329-345.

Abdel-Mawgoud, A.M., Lépine, F., Déziel, E., 2010. Rhamnolipids: diversity of structures, microbial origins and roles. Appl. Microbiol. Biotechnol. 86, 13231336.

Aparna, A., Srinikethan, G., Smitha, H., 2012. Production and characterization of biosurfactant produced by a novel Pseudomonas sp. 2B. Colloids Surf. B 95, 2329.

Benincasa, M., Accorsini, F.R., 2008. Pseudomonas aeruginosa LBI production as an integrated process using the wastes from sunflower-oil refining as a substrate. Bioresour. Technol. 99, 3843-3849.

Benincasa, M., Abalos, A., Oliveira, I., Manresa, A., 2004. Chemical structure, surface properties and biological activities of the biosurfactant produced by Pseudomonas aeruginosa LBI from soapstock. Antonie Van Leeuwenhoek 85, 1-8.

Bharali, P., Konwar, B.K., 2011. Production and physico-chemical characterization of a biosurfactant produced by Pseudomonas aeruginosa OBP1 isolated from petroleum sludge. Appl. Biochem. Biotechnol. 164, 1444-1460.

Cha, M., Lee, N., Kim, M., Kim, M., Lee, S., 2008. Heterologous production of Pseudomonas aeruginosa EMS1 biosurfactant in Pseudomonas putida. Bioresour. Technol. 99, 2192-2199.

Dubois, M., Gilles, K.A., Hamilton, J.K., Rebers, P.A., Smith, F., 1956. Colorimetric method for determination of sugars and related substances. Anal. Chem. 28, 350-356.

Folch, J., Lees, M., Stanley, G.H.S., 1957. A simple method for the isolation and purification of total lipides from animal tissues. J. Biol. Chem. 226, 497-509.

George, S., Jayachandran, K., 2009. Analysis of rhamnolipid biosurfactants produced through submerged fermentation using orange fruit peelings as sole carbon source. Appl. Biochem. Biotechnol. 158, 694-705.

George, S., Jayachandran, K., 2013. Production and characterization of rhamnolipid biosurfactant from waste frying coconut oil using a novel Pseudomonas aeruginosa D. J. Appl. Microbiol. 114, 373-383.

Grand View Research, 2014. Biosurfactants market by product (rhamnolipids, sophorolipids, MES, APG, sorbitan esters, sucrose esters) expected to reach USD 2308.8 Million by 2020. <http://www.grandviewresearch.com/industryanalysis/biosurfactants-industry> (accessed 25.07.2014).

Gudiña, E.J., Teixeira, J.A., Rodrigues, L.R., 2010. Isolation and functional characterization of a biosurfactant produced by Lactobacillus paracasei. Colloids Surf. B 76, 298-304.

Haba, E., Pinazo, A., Jauregui, O., Espuny, M.J., Infante, M.R., Manresa, A., 2003. Physicochemical characterization and antimicrobial properties of rhamnolipids produced by Pseudomonas aeruginosa 47T2 NCBIM 40044. Biotechnol. Bioeng. $81,316-322$.

Henkel, M., Müller, M.M., Kügler, J.H., Lovaglio, R.B., Contiero, J., Syldatk, C. Hausmann, R., 2012. Rhamnolipids as biosurfactants from renewable resources: concepts for next-generation rhamnolipid production. Process Biochem. 47, 1207-1219.
Hosková, M., Schreiberová, O., Jezdík, R., Chudoba, J., Masák, J., Sigler, K., Rezanka, T., 2013. Characterization of rhamnolipids produced by non-pathogenic Acinetobacter and Enterobacter bacteria. Bioresour. Technol. 130, 510-516.

Lotfabad, T.B., Shourian, M., Roostaazad, R., Najafabadi, A.R., Adelzadeh, M.R., Noghabi, K.A., 2009. An efficient biosurfactant-producing bacterium Pseudomonas aeruginosa MR01, isolated from oil excavation areas in south of Iran. Colloids Surf. B 69, 183-193.

Lotfabad, T.B., Abassi, H., Ahmadkhaniha, R., Roostaazad, R., Masoomi, F., Zahiri, H.S. Ahmadian, G., Vali, H., Noghabi, K.A., 2010. Structural characterization of a rhamnolipid-type biosurfactant produced by Pseudomonas aeruginosa MR01: enhancement of di-rhamnolipid proportion using gamma irradiation. Colloids Surf. B 81, 397-405.

Lowry, O.H., Rosebrough, N.J., Farr, A.L., Randall, R.J., 1951. Protein measurement with the Folin phenol reagent. J. Biol. Chem. 193, 265-275.

Maddipati, P., Atiyeh, H.K., Bellmer, D.D., Huhnke, R.L., 2011. Ethanol production from syngas by Clostridium strain P11 using corn steep liquor as a nutrient replacement to yeast extract. Bioresour. Technol. 102, 6494-6501.

Nie, M., Yin, X., Ren, C., Wang, Y., Xu, F., Shen, Q., 2010. Novel rhamnolipid biosurfactants produced by a polycyclic aromatic hydrocarbon-degrading bacterium Pseudomonas aeruginosa strain NY3. Biotechnol. Adv. 28, 635-643.

Pacheco, G.J., Reis, R.S., Fernandes, A.C.L.B., da Rocha, S.L.G., Pereira, M.D., Perales, J., Freire, D.M.G., 2012. Rhamnolipid production: effect of oxidative stress on virulence factors and proteome of Pseudomonas aeruginosa PA1. Appl. Microbiol. Biotechnol. 95, 1519-1529.

Patel, R.M., Desai, A.J., 1997. Biosurfactant production by Pseudomonas aeruginosa GS3 from molasses. Lett. Appl. Microbiol. 25, 91-94.

Pereira, J.F.B., Gudiña, E.J., Doria, M.L., Domingues, M.R., Rodrigues, L.R., Teixeira, J.A., Coutinho, J.A.P., 2012. Characterization by electrospray ionization and tandem mass spectrometry of rhamnolipids produced by two Pseudomonas aeruginosa strains isolated from Brazilian crude oil. Eur. J. Mass Spectrom. 18, 399-406.

Pereira, J.F.B., Gudiña, E.J., Costa, R., Vitorino, R., Teixeira, J.A., Coutinho, J.A.P., Rodrigues, L.R., 2013. Optimization and characterization of biosurfactant production by Bacillus subtilis isolates towards microbial enhanced oil recovery applications. Fuel 111, 259-268.

Perfumo, A., Rudden, M., Smyth, T.J.P., Marchant, R., Stevenson, P.S., Parry, N.J., Banat, I.M., 2013. Rhamnolipids are conserved biosurfactants molecules: implications for their biotechnological potential. Appl. Microbiol. Biotechnol. 97, 7297-7306.

Raza, Z.A., Khalid, Z.M., Banat, I.M., 2009. Characterization of rhamnolipids produced by a Pseudomonas aeruginosa mutant strain grown on waste oils. J. Environ. Sci. Health A 44, 1367-1373.

Sarachat, T., Pornsunthorntawee, O., Chavadej, S., Rujiravanit, R., 2010. Purification and concentration of a rhamnolipid biosurfactant produced by Pseudomonas aeruginosa SP4 using foam fractionation. Bioresour. Technol. 101, 324-330.

Vaz, D.A., Gudiña, E.J., Alameda, E.J., Teixeira, J.A., Rodrigues, L.R., 2012. Performance of a biosurfactant produced by a Bacillus subtilis strain isolated from crude oil samples as compared to commercial chemical surfactants. Colloids Surf. B 89, $167-174$.

Wei, Y.H., Chou, C.L., Chang, J.S., 2005. Rhamnolipid production by indigenous Pseudomonas aeruginosa J4 originating from petrochemical wastewater. Biochem. Eng. J. 27, 146-154.

Zhang, L., Pemberton, J.E., Maier, R.M., 2014. Effect of fatty acid substrate chain length on Pseudomonas aeruginosa ATCC 9027 monorhamnolipid yield and congener distribution. Process Biochem. 49, 989-995. 